\title{
Shape Optimized Design of Microwave Dielectric Resonators by Level-Set and Topology Gradient Methods
}

\author{
H. Khalil, ${ }^{1}$ S. Bila, ${ }^{1}$ M. Aubourg, ${ }^{1}$ D. Baillargeat, ${ }^{1}$ S. Verdeyme, ${ }^{1}$ F. Jouve, ${ }^{2}$ \\ C. Delage, ${ }^{3}$ T. Chartier ${ }^{4}$ \\ 1 XLIM UMR 6172, Université de Limoges/CNRS, 123 avenue Albert Thomas, 87060 Limoges, France \\ 2 Laboratoire Jacques-Louis Lions, UMR 7598, Université Paris Diderot, F-75005 Paris, France \\ ${ }^{3}$ CTTC, Parc Ester Technopole, rue Soyouz, 87068 Limoges, France \\ 4 SPCTS UMR 6638, Université de Limoges/CNRS, 47 à 73 avenue Albert Thomas, 87065 Limoges, \\ France
}

Received 8 April 2009; accepted 14 June 2009

\begin{abstract}
The finite element method is coupled with the topology gradient (TG) and levelset (LS) methods for optimizing the shape of microwave components using a computer-aided design model. On the one hand, the LS approach is based on the classical shape derivative; while on the other hand, the TG method is precisely designed for introducing new perturbations in the optimization domain. These two approaches, which consist in minimizing a cost function related to the component behavior, are first described. Regarding given electrical specifications, these techniques are applied to optimize the distribution of ceramic parts of a dual-mode resonator in order to improve its behavior. The optimized dielectric resonators result in a wide spurious-free stop band. A comparison between classical and optimized dual mode resonator is presented. Theoretical results are then validated by careful measurements. (c) 2009 Wiley Periodicals, Inc. Int J RF and Microwave CAE 20: 33-41, 2010.
\end{abstract}

Keywords: shape optimization; topology gradient; level-set; microwave components; finite element method

\section{INTRODUCTION}

Shape optimization methods [1-8] are well suited for determining the shape of an object in order to satisfy given specifications. Shape optimization strategies can be mainly classified into two categories: boundary optimization, which modifies the contour of the object as done by the level-set (LS) method, and topology optimization, which introduces a local perturbation within the domain as done by the topology gradient (TG) method.

In both cases, optimization of the numerical model is achieved thanks to a gradient evaluation calculated for a cost function related to the model behavior. Such numerical problems are generally constrained ones, which require the resolution of an adjoint problem, similarly to a geo-

Correspondence to: H. Khalil; e-mail: hassan.khalil@xlim.fr DOI 10.1002/mmce.20400

Published online 10 November 2009 in Wiley InterScience (www.interscience.wiley.com). metrical design sensitivity approach [9]. However, considering shape optimization methods, the essential difference lies in the number of variables, which is often huge.

The LS method $[10,11]$ is known for modeling propagating fronts and is based on the shape derivative, moving the boundary along the gradient direction during the optimization process. LS methods have been applied very successfully in many areas of scientific modeling and optimization [12-15] and more recently, in the design of microwave components [16] but generally in two-dimensional problems.

The TG method evaluates a gradient concerning a small modification of the domain, translated into adding or removing material. TG optimization was originally developed for mechanics design problems, and it has been recently adapted to other types of design problems, such as electrical and electronic devices [17-19].

The TG method coupled with a finite element method (FEM) for optimizing microwave components has been

(C) 2009 Wiley Periodicals, Inc. 
demonstrated in 2D [20] for optimizing the distribution of metal upon the surface of microstrip components and in 3D [21] for optimizing a dual-mode dielectric resonator.

In this article, the previous work is extended with the addition of the LS approach for the optimized design of microwave dielectric resonators (3D problem). A geometry optimization is first applied to design a dielectric resonator filter. For improving the performance of the microwave component, the shape and the topology are modified applying respectively the LS and the TG methods.

Optimization strategies are then compared and optimized structures are manufactured and measured for experimental verification.

\section{SHAPE OPTIMIZATION METHODS COUPLED WITH FINITE ELEMENTS}

\section{A. Finite Element Method}

For analyzing a microwave component with a numerical method such as finite elements, the electromagnetic model is discretized into small elements before solving Maxwell's equations. Applying the finite element method, the following matrix equation is solved:

$$
A(\eta) E(\eta)=B
$$

where $A$ is a square symmetric matrix (Maxwell's operator) representing the geometrical and material properties of the discretized model, $B$ is the column vector of imposed sources, and $E$ the unknown field vector, solution of eq. (1).

\section{B. TG Method}

The TG optimization procedure starts from an initial configuration of the domain and converges iteratively to the optimum shape using the gradient calculation. The later gradient is calculated at the first order by analyzing the sensitivity of the cost function with respect to a small perturbation of the domain $\Omega$.

We consider $\eta$ as the reference perturbation centered at origin. The perturbation centered at point $x$ and of size $h$, is characterized by:

$$
\eta_{x, h}=x+h \eta
$$

For calculating the gradient, the principle of topological asymptotic is utilized [22]. The approach gives then a more precise expression of this gradient.

The main idea is to isolate the perturbation in the fixed domain $D$ (see Fig. 1). The interest of this approach is to reduce the domain of problem to $\Omega^{\prime}=\Omega \mathrm{V}$ ( $D$ excluded from $\Omega$ ). The perturbation becomes a surface condition on the contour $\Gamma . A^{0}$ is the initial Maxwell's operator (without perturbation) and $A^{x, h}$ the perturbed operator. A similar notation is utilized for the electric field $E$.

The perturbation does not depend on the imposed sources, so the problem in (1) can be divided into two parts:

$$
A_{\Omega}^{0} E_{\Omega}^{x, h}=B^{0}-B_{\Gamma}^{x, h} \quad A_{\mathrm{D}}^{x, h} E_{\mathrm{D}}^{x, h}=B_{\Gamma}^{x, h}
$$

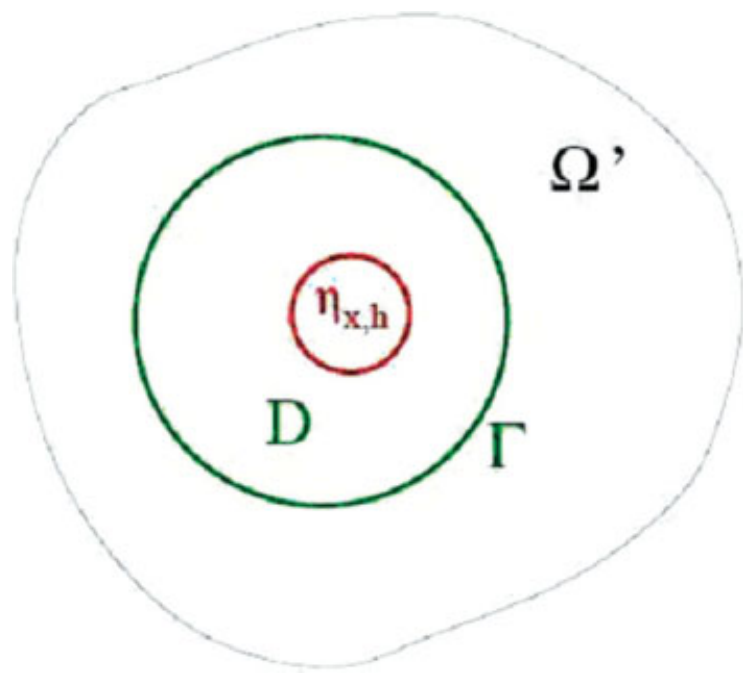

Figure 1 Perturbation in the fixed domain D. [Color figure can be viewed in the online issue, which is available at www. interscience.wiley.com.]

assuming on contour $\Gamma$ that:

$$
E_{\mathrm{D}}^{x, h} / \Gamma=E_{\Omega}^{x, h} / \Gamma
$$

When the perturbation is negligible $(h \rightarrow 0)$, the perturbed field on contour $\Gamma$ equals the initial field (without perturbation):

$$
E_{\Omega}^{x, h} / \Gamma=E_{\Omega}^{0} / \Gamma
$$

A classical approach for solving this class of problems is to minimize the cost function $J$ applying the Lagrange method where $L$, the Lagrangian function, is defined by:

$$
L\left(Y, \eta_{x, h}, P\right)=J\left(E^{x, h}\right)+\operatorname{Re} \operatorname{Tr}\left(P^{*}\left(A^{x, h} Y-B\right)\right)
$$

where $\operatorname{Tr}$ is the trace function, which is defined by the sum of the diagonal elements of a matrix.

In our case, $Y$ is replaced by $E$, solution of (1):

$$
J\left(E^{x, h}\right)=L\left(E, \eta_{x, h}, P\right), \forall P
$$

$P$ is the vector of Lagrange multipliers, an independent parameter that converts the constrained problem into an unconstrained one. The TG is calculated by the difference between the perturbed cost function and the initial cost function (without perturbation):

$$
g_{x, h}=J\left(E^{x, h}\right)-J\left(E^{0}\right)+\operatorname{Re} \operatorname{Tr}\left(P^{*}\left(A^{x, h} E^{x, h}-A^{0} E^{0}\right)\right)
$$

If $J$ is a linear function of field $E$, one can write:

$$
\begin{aligned}
g_{x, h}=\operatorname{Re}\left(\left(\partial_{\mathrm{E}} J\left(E^{0}\right)\right)^{T}+\right. & \left.P^{*} A^{0}\right)\left(E^{x, h}-E^{0}\right) \\
& +\operatorname{Re} \operatorname{Tr}\left(P^{*}\left(A^{x, h}-A^{0}\right) E^{x, h}\right)
\end{aligned}
$$




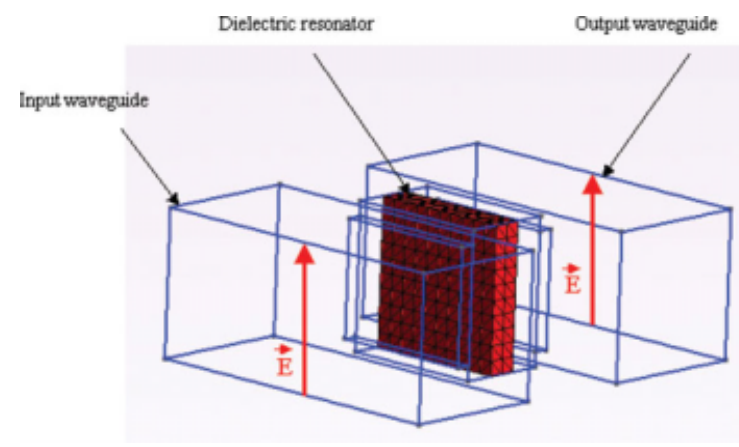

Figure 2 Square dielectric resonator included in a cavity. [Color figure can be viewed in the online issue, which is available at www.interscience.wiley.com.]

$P$ is then the solution of an adjoint problem:

$$
\left(A^{0}\right)^{*} P=-\partial_{\mathrm{E}} J^{*}\left(E^{0}\right)
$$

The TG can be finally calculated as follows:

$$
g_{x, h}=\operatorname{Re} \operatorname{Tr}\left(P^{*}\left(A_{\mathrm{D}}^{x, h}=A_{\mathrm{D}}^{0}\right) E_{\mathrm{D}}^{x, h}\right)
$$

One can note that, in the previous equations, superscripts $T$ and * denote, respectively the transposition and conjugation operators.

After solving the direct and adjoint problems successively, the TG is calculated for each element in the fixed domain $D$, then all topological elements with a negative gradient can be modified to minimize the cost function, and the TG can be then evaluated with the new configuration. The procedure is repeated iteratively until a local minimum is attained.

\section{LS Method}

The variables are defined on the contour of the object $\Omega$. It suffices to mesh $\Omega$ and to deform the contour $\eta$ according to the descent direction $\theta$. Let a bounded domain $D \subset R^{d}\left(R^{d}\right.$ is an Euclidean space with $d=2$ or 3$)$ be the working domain in which all admissible shapes $\Omega$ are included, $\Omega(D$. In numerical practice, the domain $D$ will be uniformly meshed once and for all. The boundary of $\Omega$ is parameterized by means of a LS function, following the idea of Osher and Sathian [11]. This LS function $\psi$ is defined in $D$ such that

$$
\left\{\begin{array}{l}
\psi(x)=0 \quad \Leftrightarrow \quad x \in \partial \Omega \cup D, \\
\psi(x)<0 \quad \Leftrightarrow \quad x \in \Omega, \\
\psi(x)>0 \quad \Leftrightarrow \quad x \in(D \backslash \bar{\Omega}) .
\end{array}\right.
$$

To minimize $J$, a shape derivative is computed so that:

$$
J^{\prime}(\Omega)(\theta)=\int_{\partial \Omega} f(E, P, \eta) \theta \cdot \mathrm{nds}
$$

where $f(E, P, \eta)$ is a scalar function, which depends on $E$, $P$ and $\eta$.
Therefore, we can define a descent direction in the whole domain $D$ by:

$$
\theta=-f \cdot n
$$

where $n$ is the normal to the shape $\Omega$.

The evolution of the LS function is governed by the following Hamilton-Jacobi transport equation:

$$
\frac{\partial \psi}{\partial t}-f|\nabla \psi|=0
$$

Transporting $\psi$ by [15] is equivalent to move the boundary of $\Omega$ (the zero LS of $\psi$ ) along the descent gradient direction- $J^{\prime}(\Omega)$.

\section{APPLICATIONS}

Previous shape optimization methods are applied to the design optimization of dielectric resonator filters.

Dielectric resonator filters are widely used in communication systems [23] due to their high selectivity, small size and mass. The square dielectric resonator, shown in Figure 2, is introduced in a metallic cavity, in contact with the walls. The dielectric resonator is excited with coupling irises connected to standard rectangular waveguides.

Because of its symmetry, the resonator could support two degenerate modes (polarizations) in order to form a dual mode filter. Turning the iris and the waveguide at one end by $90^{\circ}$ as shown in Figure 3, the two polarizations can be coupled together, in order to form a 2-pole filter if the symmetry of the resonator is perturbed.

The structure in Figure 3 is the initial configuration for designing the filter. The design is achieved by geometry optimization and then by TG and LS methods.

\section{A. Design by Geometry Optimization}

A square corner is cut within the dielectric resonator, as shown in Figure 4, in order to couple the two

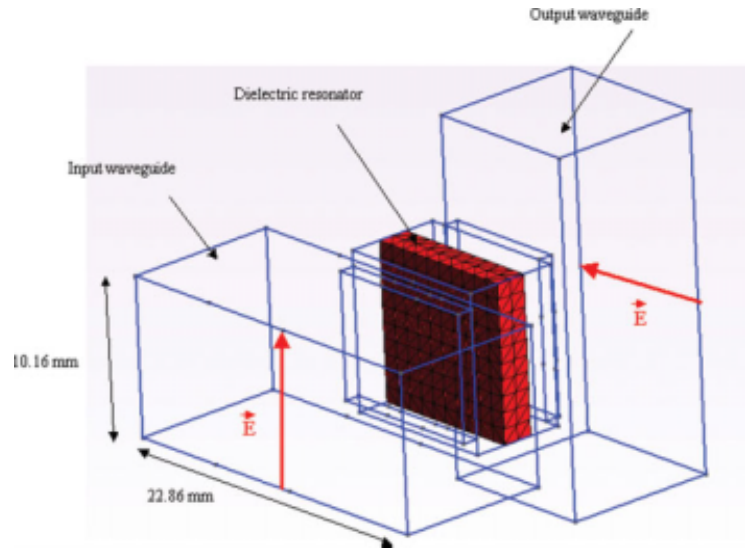

Figure 3 Initial dielectric resonator configuration for geometry and shape optimizations. [Color figure can be viewed in the online issue, which is available at www. interscience.wiley.com.] 


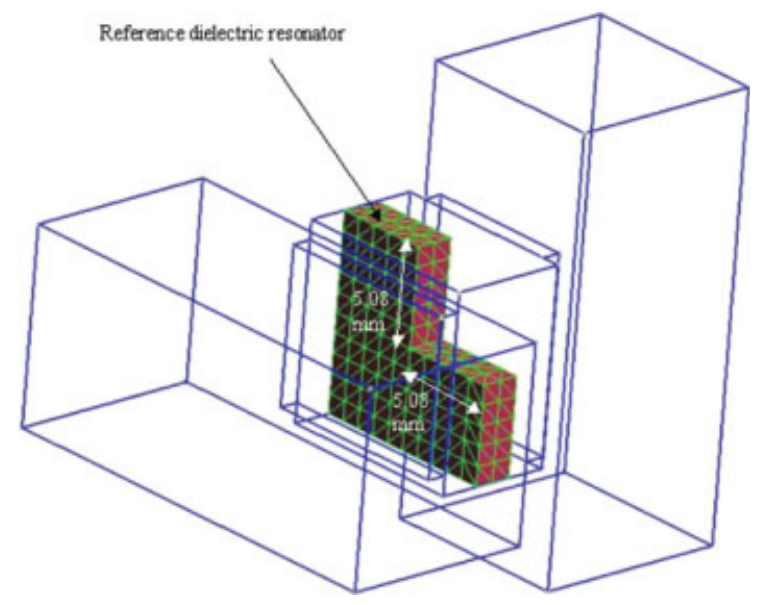

Figure 4 Dual-mode dielectric resonator filter designed applying geometry optimization. [Color figure can be viewed in the online issue, which is available at www. interscience.wiley.com.]

polarizations. The dimensions of the square cut are then adjusted by geometry optimization. A two-pole filter is then designed and the optimized scattering parameters $(S)$ are shown in Figure 5.

The scattering parameters present a $270 \mathrm{MHz}$ passband at $10 \mathrm{GHz}$ and a parasitic resonance at $12.5 \mathrm{GHz}$. The parasitic resonance cannot be attenuated nor pushed upward in frequency because of lack of parameters. Indeed, geometry optimization modifies the dimensions of the structure but is not able to modify the shape or the topology.

Using TG and LS algorithms, the optimization goal is now to recover the bandwidth at $10 \mathrm{GHz}$ with similar characteristics and while attenuating or shifting the parasitic resonance at $12.5 \mathrm{GHz}$.

\section{B. Design by TG Optimization}

The initial configuration for TG optimization is the dielectric resonator shown in Figure 3. The cost function to be minimized measures the distance to scattering parameters optimized by geometry optimization (see Fig. 5) for fre-

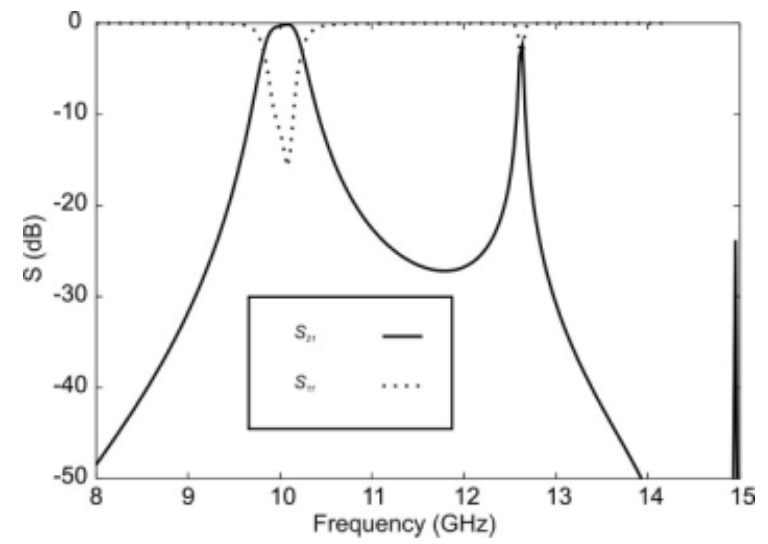

Figure 5 Scattering parameters of the dual-mode dielectric resonator filter designed by geometry optimization (see Fig. 4).

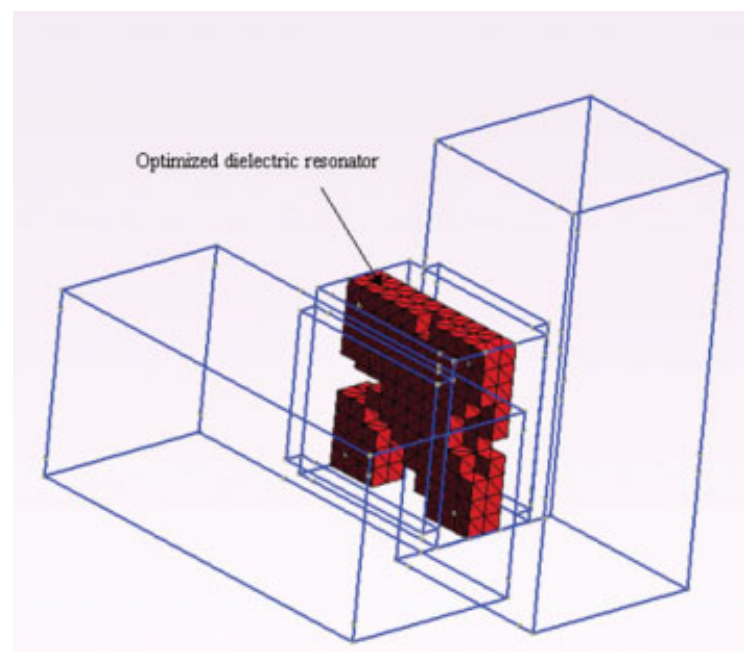

Figure 6 Dual-mode dielectric resonator filter designed applying TG optimization. [Color figure can be viewed in the online issue, which is available at www.interscience. wiley.com.]

quencies between 8 and $10.5 \mathrm{GHz}$. For higher frequencies, the cost function is simply defined by a threshold. A practical formulation can be found in [21].

The state of a topological element is defined by its dielectric permittivity $(\eta=0$ for $\varepsilon r=1, \eta=1$ for $\varepsilon r=$ 8.945). The optimization domain, which contains the initial resonator, is discretized into 200 topological elements. All elements are set to state $\eta=1$ as initial condition, in accordance with the initial configuration.

After each gradient calculation, a portion of the elements having a gradient that lowers the cost function are modified by destruction $(\eta=1 \rightarrow 0)$ or construction $(\eta=$ $0 \rightarrow 1)$ of the dielectric material. Convergence is achieved after 13 iterations when no more modification on the elements could minimize the cost function.

After optimization, one can notice from Figure 6 that the optimized dielectric resonator differs broadly from the resonator designed by geometry optimization (see Fig. 4). In Figure 7, a comparison of scattering parameters

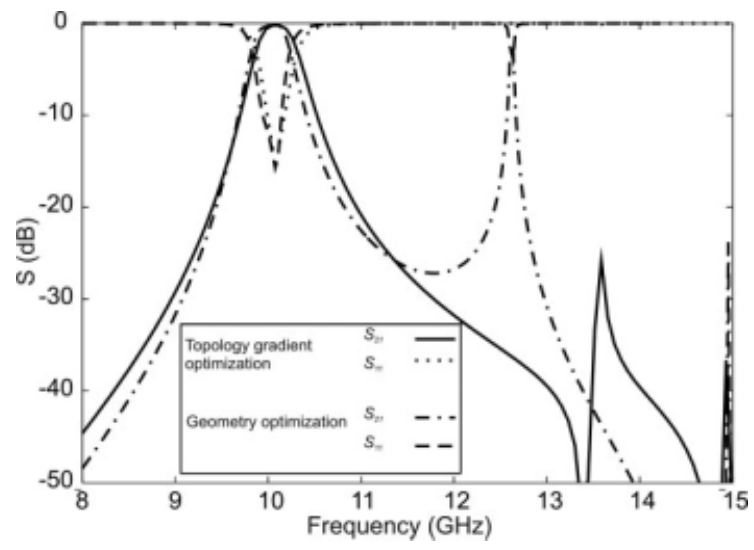

Figure 7 Scattering parameters of the dual-mode dielectric resonator filter designed by TG optimization (see Fig. 6). 


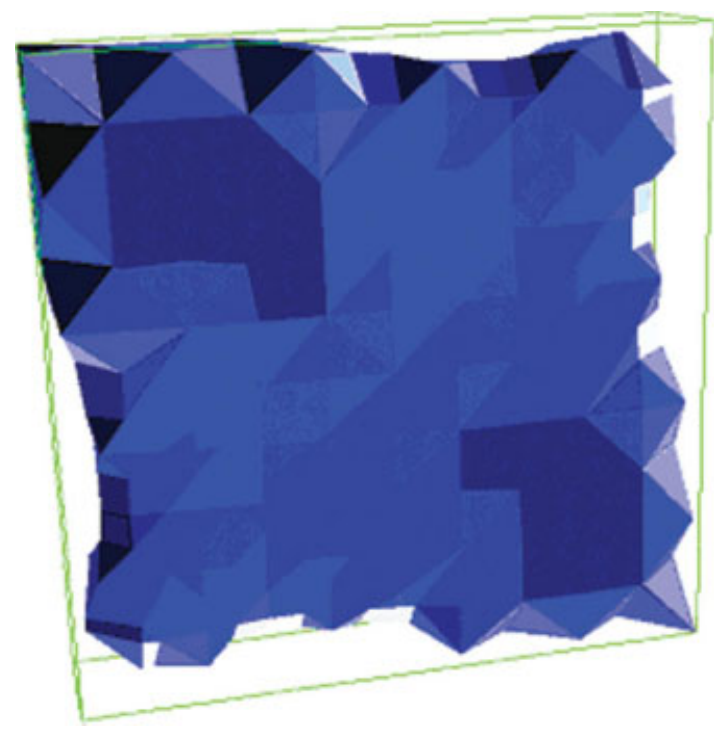

Figure 8 Optimal dielectric resonator obtained by LS method. [Color figure can be viewed in the online issue, which is available at www.interscience.wiley.com.]

between optimized structures is presented. One can clearly note that the passband at $10 \mathrm{GHz}$ is recovered, while the parasitic resonance at $12.5 \mathrm{GHz}$ is shifted to a higher frequency and attenuated to $-25 \mathrm{~dB}$.

\section{Design by LS Optimization}

The initial configuration, the mesh discretization and the cost function to be minimized are identical to those used with the topology gradient.

The shape is defined by the LS function with $\psi<0$ inside the dielectric object and $\psi>0$ outside. The whole domain is defined initially with $\psi<0$ according to the initial configuration in Figure 3.

The shape is deformed iteratively through the transport of $\psi$ given in [15]. The convergence is achieved when no more modification can decrease the cost function.

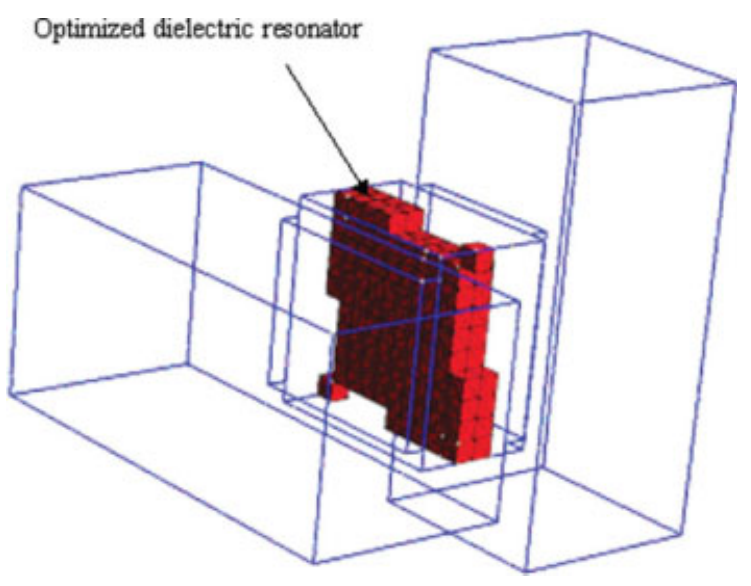

Figure 9 Dual-mode dielectric resonator filter designed applying LS optimization, after approximation. [Color figure can be viewed in the online issue, which is available at www.interscience.wiley.com.]

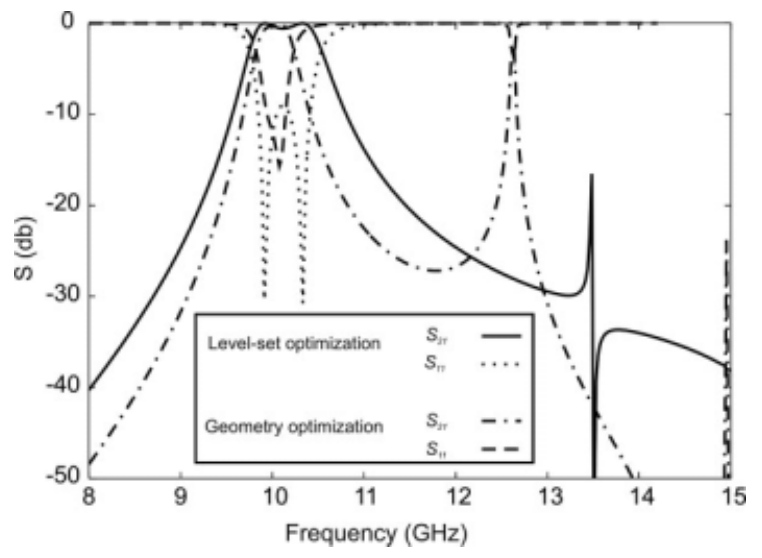

Figure 10 Scattering parameters of the dual-mode dielectric resonator filter designed by LS optimization (see Fig. 9).

The shape attained by LS optimization is given in Figure 8 . As this optimal shape is not conformal to the original mesh, the object is approximated by the shape given in Figure 9. For the approximation, each square element is filled with dielectric material when its volume contains at least $50 \%$ of dielectric material in the optimal LS shape. Scattering parameters obtained with the optimized resonator (after approximation) are given in Figure 10.

One can note that the parasitic resonance is attenuated and shifted again compared to geometry optimization. However, convergence is not achieved for the passband characteristic. Indeed, the bandwidth is larger than expected because of the difference between optimized and approximated forms. Such a discrepancy could be reduced using a smaller mesh discretization or parameterizing the geometry.

In the next section, an iterative coupling between LS and TG methods is proposed. Both methods are gradienttype algorithms, so our coupled method can be cast into the framework of alternate directions descent algorithms.

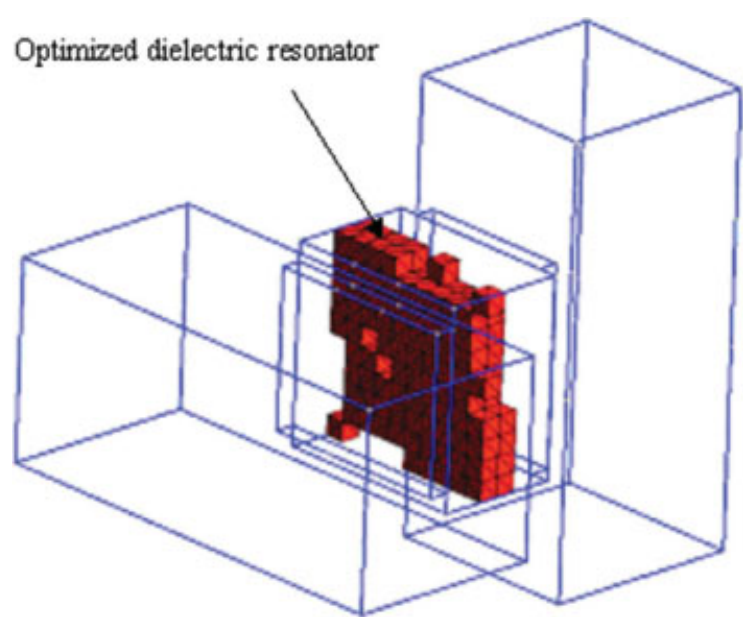

Figure 11 Dual-mode dielectric resonator filter designed applying coupled LS and TG optimization. [Color figure can be viewed in the online issue, which is available at www.interscience.wiley.com.] 


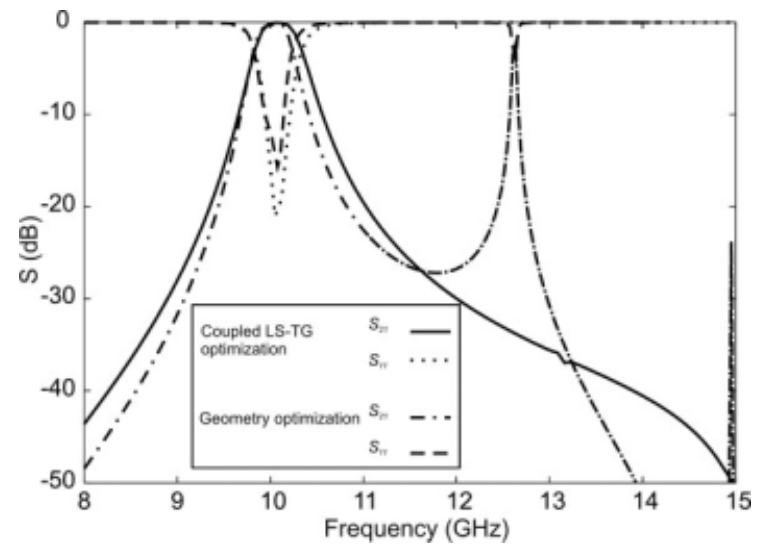

Figure 12 Scattering parameters of the dual-mode dielectric resonator filter designed by coupled LS and TG optimization (see Fig. 11).

\section{Design by Coupled LS and TG Optimization}

The LS method relies on the shape derivative while the TG method is based on a perturbation of the domain. These two algorithms define independent descent directions that can be simply alternated.

The optimization program starts with the LS algorithm, deforming the shape as far as the algorithm converges. The optimization program continues with the TG algorithm for creating or removing material locally as long as the cost function decreases. The two algorithms are alternatively applied for optimizing the dielectric resonator until the two algorithms cannot converge any more.

Using the resonator designed by LS optimization in Figure 9, the TG is applied. After six iterations, the optimal resonator is found as presented in Figure 11. Applying the LS algorithm, the optimization does not converge anymore.

One can note from Figure 12 that the passband is correctly obtained and that the parasitic resonance at 12.6 $\mathrm{GHz}$ is eliminated.

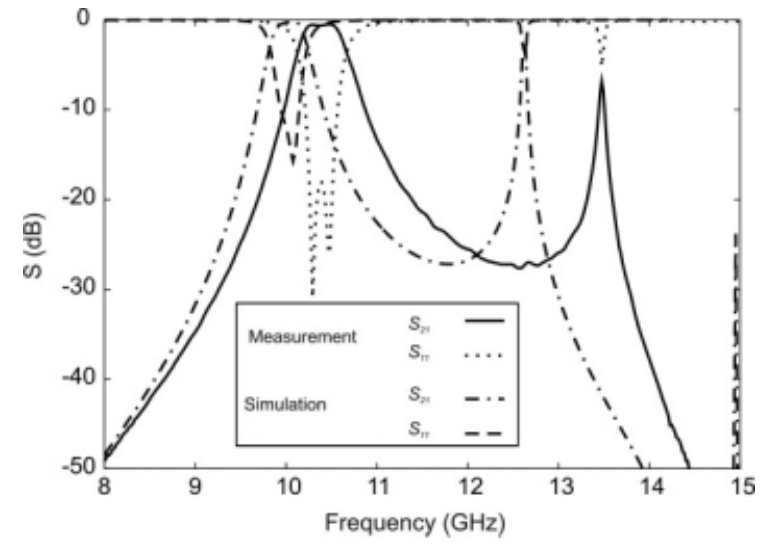

Figure 13 Measured scattering parameters for the dual-mode dielectric resonator filter designed by geometry optimization.

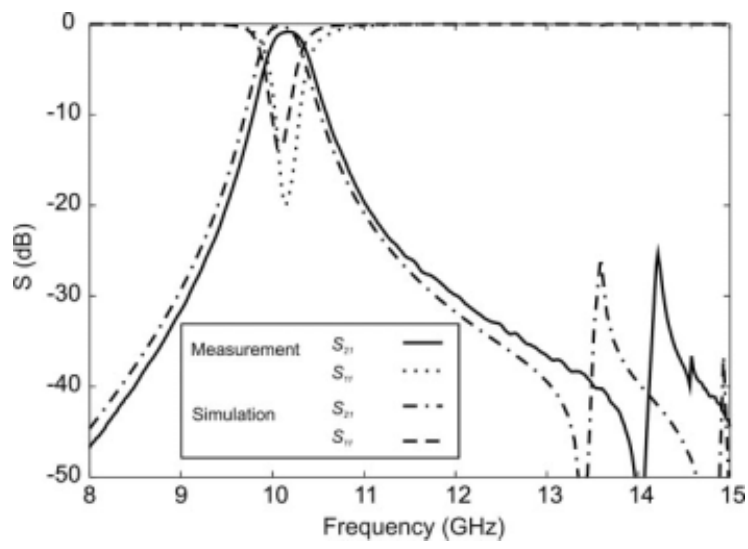

Figure 14 Measured scattering parameters for the dual-mode dielectric resonator filter designed by TG optimization.

\section{EXPERIMENTS}

Optimized dielectric resonators are fabricated by ceramic stereo-lithography [24], which is a rapid prototyping process able to realize complex ceramic objects. The resonators are then positioned within a metallic cavity and connected to standard waveguides for $S$-parameters measurement.

Figures 13-15 show a comparison between simulated and measured scattering parameters for dielectric resonators designed respectively by geometry optimization, TG optimization and coupled LS-TG optimization.

One can note that measured $S$-parameters are shifted to higher frequencies, especially for the resonator designed by geometry optimization. The shift is mainly due to a small discrepancy in the thickness of manufactured resonators. Fabrication and measurement have been performed without preliminary tuning, but such a discrepancy could be corrected by a more accurate control of the thickness and/or by using tuning elements such as screws within the cavities.

However, the measured passband characteristic and parasitic resonance are generally found with a good

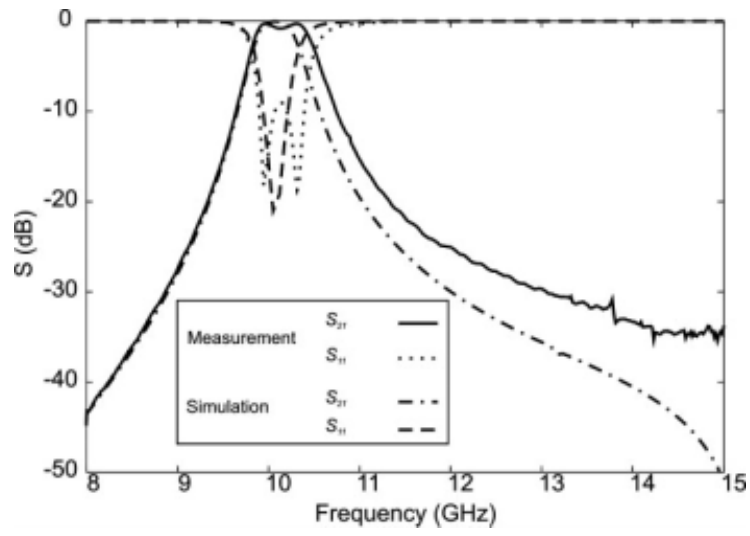

Figure 15 Measured scattering parameters for the dual-mode dielectric resonator filter designed by coupled LS and TG optimization. 
agreement compared to simulations, confirming the ability of such shape optimization methods for the advanced design of microwave components.

\section{v. CONCLUSIONS}

This article presents an original shape optimization approach, based on finite element modeling, for improving the behavior of dual-mode dielectric resonator filters.

A first design is achieved applying a geometrical optimization, but this classical approach is not sufficient for eliminating parasitic resonances occurring at higher frequencies. LS and TG methods are applied respectively for modifying the contour and for creating new perturbations in the dielectric resonator in order to improve the stop band performance.

These two algorithms are local optimization methods with independent descent directions. The best result is obtained when the two algorithms are coupled together, leading to an original shape optimization scheme, capable of both moving the boundary and creating holes within the object.

Numerical designs are finally validated by careful measurements, showing a good agreement, and confirming the potential application of such shape optimization methods for the advanced design of dielectric resonator filters.

An extension of this work is its application to unloaded quality factor optimization for the design of dielectric resonator filters with improved loss performances.

\section{ACKNOWLEDGMENT}

This work is supported by the French Research Agency (ANR DOPROCOF).

\section{REFERENCES}

1. J. Sokolowski and A. Zochowski, On the topological derivative in shape optimization, SIAM J Control Optim 37 (1999), 1251-1272.

2. W. Nadir, Y.I. Kim, and O.L. de weck, Structural shape optimization considering both performance and manufacturing cost, Collect Tech Pap-10th AIAA/ISSMO Multidiscip Anal Optim Conf 5 (2004), 3240-3251.

3. G. Allaire and F. Jouve, Optimal design of micro-mechanisms by the homogenization method, Eur J Finite Elem 11 (2002), 405-416.

4. P. Mader, M. Masmoudi, and C. Mangenot, Topological asymptotic for waveguide optimization, IEEE Antenn Propag Soc, AP-S Int Symp (Digest) 1 (2001), 616-619.

5. A. Kozak and K. Gwarek Wojciech, Unrestricted arbitrary shape optimization based on 3D electromagnetic simulation, IEEE MTTS Int Microwave Symp Dig 1 (1998), 17-20.

6. J.K. Byun, I.H. Park, W. Nah, J.H. Lee, and J. Kang, Comparison of shape and topology optimization methods for HTS solenoid design, IEEE Trans Appl Supercond 14 (2004), $1842-1845$

7. H. Choo and H. Ling, Design of broadband and dual-band microstrip antennas on a high-dielectric substrate using a genetic algorithm, IEE Proc: Microwaves Antennas Propag 150 (2003), 137-142.
8. A. Kido, H. Deguchi, M. Tsuji, and M. Ohira, Multi-resonator generation in arbitrarily-shaped planar circuit filters by genetic optimization, Proc 37th Eur Microwave Conf (EuMC), Munich, Germany, 2007, pp. 1241-1244.

9. H. Akel and J.P. Webb, Design sensitivities for scatteringmatrix calculation with tetrahedral edge elements, IEEE Trans Magn 36 (2000), 1043-1046.

10. G. Allaire, F. de Gournay, F. Jouve, and A.M. Toader, Structural optimization using topological and shape sensitivity via a level set method, Control Cybern 34 (2005), 59-81.

11. S. Osher and J.A. Sethian, Fronts propagating with curvature dependent speed: Algorithms based on Hamilton-Jacobi Formulations, J Comput Phys 79 (1988), 12-49.

12. J.S. Osher and F. Santosa, Level set method for optimization problems involving geometry and constraints. I. frequencies of a two-density inhomogeneous drum, J Comput Phys 171 (2001), 272-288.

13. J.A. Sethian and A. Wiegmann, Structural boundary design via level set and immersed interface methods, J Comput Phys 163 (2000), 489-528.

14. J.S. Suri, K. Liu, S. Singh, S.N. Laxminarayan, X. Zeng, and L. Reden, Shape recovery algorithms using level sets in 2-D/ 3-D medical imagery; A state-of-the-art review, IEEE Trans Inf Technol Biomed 6 (2002), 8-28.

15. G. Allaire, F. Jouve, and A.M. Toader, Structural optimization by the Level-set method, C R Acad Sci Paris, ser I 334 (2002), 1125-1130.

16. M.R. Hajihashemi and M. El-Shenawawee, Shape reconstruction using the Level Set method for microwave applications, IEEE Antennas Wireless Propag Lett 7 (2008), 92-96.

17. J.K. Byun, S.Y. Hahn, and H. Park, Topology optimization of electrical devices using mutual energy and sensitivity, IEEE Trans Magn 35 (1999), 3718-3720.

18. Y.S. Chung and C. Cheon, Optimal shape design of dielectric structure using FDTD and topology optimization, IEEE MTTS Int Microwave Symp Dig 1 (2001), 2063-2066.

19. J.K. Byun and H. Park, Design of dielectric waveguide filters using topology optimization technique, IEEE Trans Magn 43 (2007), 1573-1576.

20. A. Assadihaghi, S. Bila, C. Durousseau, D. Baillargeat, M. Aubourg, S. Verdeyme, M. Rochette, J. Puech, and L. Lapierre, Design of microwave components using topology gradient optimization, Proc 36th Eur Microwave Conf (EuMC), Manchester, United Kingdom, art. no. 4057850, 2006, pp. 462-465.

21. H. Khalil, N. Delhote, S. Bila, M. Aubourg, S. Verdeyme, J. Puech, L. Lapierre, C. Delage, and T. Chartier, Topology gradient optimization applied to the design of a dual-mode filter including a dielectric resonator, IEEE MTTS Int Microwave Symp Dig, art. no. 4633035, Atlanta, GA, 2008, pp. 1381-1384.

22. M. Masmoudi, J. Pommier, and B. Samet, The topological asymptotic expansion for the Maxwell equations and some application, Inverse Probl (Institute of Physics publishing) 21 (2005), 547-564.

23. A. Deleniv and S. Gevorgian, Design of the compact bandpass filter utilizing dielectric loaded waveguide resonator, Proc 37th Eur Microwave Conf (EuMC), Munich, Germany, art. no. 4405335, 2007, pp. 886-889.

24. C. Duterte, N. Delhote, D. Baillargeat, S. Verdeyme, Y. Abouliatim, and T. Chartier, 3D ceramic microstereolithography applied to sub-millimeter devices manufacturing, Proc 37th Eur Microwave Conf (EuMC), Munich, Germany, 2007, pp. 814-817. 


\section{BIOGRAPHIES}

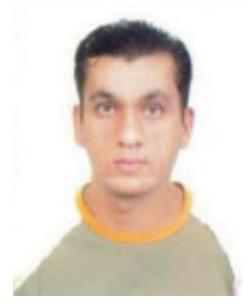

Hassan Khalil was born in Lebanon, in 1982. He received the Master's degree in High Frequency and Optical Telecommunications from the University of Limoges, Limoges, France, and is currently working toward the Ph.D. degree with XLIM, research laboratory of the University of Limoges. His research interests are dedicated to shape optimization of RF and microwave components.

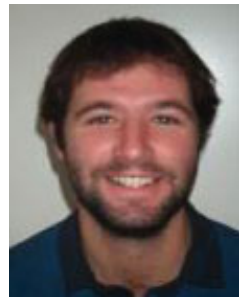

Stéphane Bila was born in Paris, France, in September 1973. In 1999, he received the Ph.D. degree from the University of Limoges, Limoges, France. He then held a post-doctoral position during one year with the French Space Agency (CNES), Toulouse, France. In 2000, he became a researcher at the French National Centre for Scientific Research (CNRS) and joined IRCOM (now XLIM), Limoges, France. His research interests include numerical modeling, optimization and computer aided techniques for the advanced synthesis of microwave components and circuits.

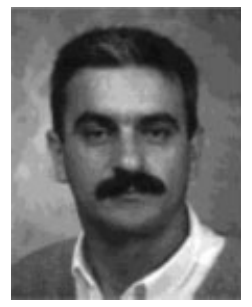

M. Aubourg was born in Neuvy Saint-Sepulcre, France, in April 1950. $\mathrm{He}$ received the Maitrise of Mathematics, the Doctorat de troisième cycle and the Doctorat d'état degrees from the University of Limoges, Limoges, France, in 1975, 1978, and 1985, respectively. Since 1979, he has been Chargé de Recherche at the CNRS, working at XLIM (formerly IRCOM), University of Limoges. His main area of interest is the application of the FEM in the design microwave transmission components.

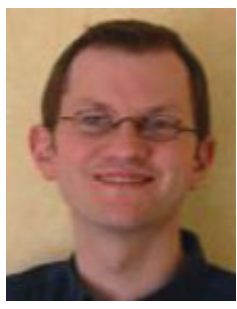

Dominique Baillargeat was born in Le Blanc, France, in 1967. He received the $\mathrm{Ph} . \mathrm{D}$. degree in 1995 from the Research Institute in Microwave and Optical Communications (IRCOM) from the University of Limoges, France. From 1995 to 2005, he was associate professor with the Microwave Circuits and Devices Team from the IRCOM laboratory. He is currently professor, and his fields of research concern the development of methods of design for microwave devices. These methods include CAD techniques based on hybrid approach coupling electromagnetic, circuits, and thermal analysis, synthesis and electromagnetic optimization techniques, etc. They are mainly dedicated to the packaging of millimeter wave and optoelectronics modules, and to the design of millimeter original filters based on new topologies, concepts (EBG) and/ or technologies (Silicon, LTCC, etc.).

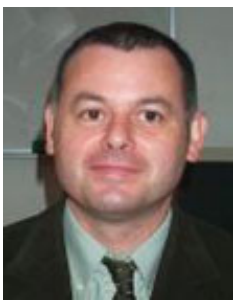

Serge Verdeyme was born in Meilhards, France, in June 1963. He received the Doctorat degree from the University of Limoges, Limoges, France, in 1989. He is currently Professor at the Research Institute on Microwave and Optical Communications (IRCOM), University of Limoges, and Head of the team Microwave Circuits and Devices. His main area of interest concerns the design and the optimization of microwave devices. $\mathrm{He}$ is a member of IEEE.

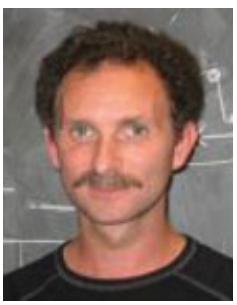

François Jouve was born in Paris, France, in 1963. He is a former student of the Ecole Normale Supérieure (Paris). He received his Ph.D. in mathematics of the University Paris 6 in 1992. He became researcher at the French National Centre for Scientific Research (CNRS) in 1995 and joined the University Paris Diderot (Paris 7) in 2006 as professor. He is a specialist of numerical methods and scientific computing, especially in the field of shape and topology optimization. 


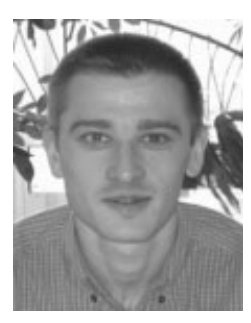

Cyrille Delage was born in $\mathrm{La}$ Rochefoucauld-France, in 1974, ceramic engineer from Ecole Nationale Superieure de Ceramique Industrielle (ENSCI), is in charge of research programs on stereolithography process applied on ceramic materials. He has 7 years of experience.

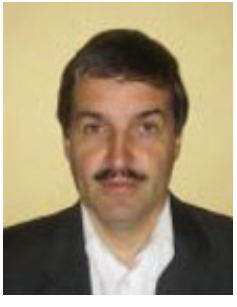

Thierry Chartier is graduated from ENSCI (Ceramic) Engineering School in Limoges, France, in 1982 and received the Ph.D. degree from University of Limoges in 1985. He his currently CNRS Research Director and Director of the Science of Ceramic Processes and Surface Treatments Laboratory associated with CNRS (SPCTSUMR CNRS 6638). His research mainly concerns the development of new ceramic shaping methods and the achievement of specific parts with controlled microstructures and designs. T. Chartier is the author/coauthor of 105 reviewed papers. 